\title{
Carrying the torch \& facing new challenges
}

\author{
Haidong Dong ${ }^{1}$
}

Published online: 3 November 2020

(c) Springer-Verlag GmbH Germany, part of Springer Nature 2020

More than 60 years ago, while the field of cancer immunology was in its advent searching for a novel therapy for cancer, Dr. Georges Mathé, the founding editor of Cancer Immunology, Immunotherapy (CIIM), performed the world's first successful allogeneic bone marrow transplant (1958), which he considered to be a form of cancer therapy and sparked a light in the dark for researchers. Years later in 1972, he lit the torch in the form of a specialist journal of CIIM, a title that perfectly reflected his foresight for the potential impact of immunotherapy in cancer treatment now widely accepted and practiced 40 years later.

CIIM is recognized as the first journal in the cancer immunology field and it is important that we pay homage to the founder, and the successor pioneers, as we stand on their shoulders continuing the progress and advancements in this field of research and treatment. We humbly recognize the contribution and leadership of the founder and previous editors: Founding Editor: Georges Mathé; and previous CoEditors-in-Chief: Robert Baldwin, Enrico (Henry) Mihich, Graham Pawelec, and Suzanne Ostrand-Rosenberg, CIIM's visionary leaders. They each made a significant impact in cancer immunology from their own research, while leading, managing and promoting CIIM from a specialist journal to one with a general impact in cancer therapy. As one of the first journals in cancer immunology and immunotherapy, CIIM was poised to promote the field when "immunological intervention as therapeutic modality for cancer was only a concept". From there, Dr. Ostrand-Rosenberg predicted that "immunotherapy can become an efficacious mainstream treatment for cancer" in 2013. When Dr. Pawelec assumed the role of EIC of CIIM (2003), he said: "While the time may be propitious for the maturing field of cancer immunology and immunotherapy, times are difficult for small specialist journals like this one...It is my firm belief that

Haidong Dong

dong.haidong@mayo.edu

1 Mayo Clinic College of Medicine and Science, Mayo Clinic, Rochester, MN, USA immunology is the key to successful cancer therapy.". As they predicted, the recent successful implementation of cancer immunotherapy as a standard of care in cancer treatment, reflects the importance of the research published in CIIM and other journals, that collectively contribute to the establishment of cancer immunotherapy along with other cancer therapies as standard of care.

This is an auspicious time to celebrate their vision, leadership, and many accomplishments in CIIM. As Dr. Pawelec pointed out: "Translational research, and with it the publication not only of success stories, but also of the less positive data, will become of ever greater importance in this difficult area". Under their leadership, commitment and service, the research articles in CIIM contributed to the collective wisdom of great minds around the world for the breakthroughs in cancer immunotherapy. A way to think about the influence of CIIM under their leadership is how often papers are read at CIIM. The increasing use of the journal's website by readers allows us a quantitative window. In 2019, there were 425,640 downloads and many readers have praised the journal for its readability and practical application of its articles in both research and clinical studies. Since the impact factor (IF) is useful for clarifying the significance of absolute citation frequencies, the IF of CIIM increased to 5.442 (2019) along with a 5 year average of IF of 4.781 .

Carrying the torch from our previous editors, this editorial team, established this year, advances-increasing the impact of CIIM, dedicated to publishing papers from around the world, including work performed in under-represented regions, and research topics with geographic impact. We are pleased to welcome the growing submissions from around the world hereafter. We truly appreciate everyone's contribution to CIIM where the fruits of researchers' efforts are published, so that cancer patients receive improved immunotherapy. To that end, in the era of evidence-based medicine, we expect more high-quality, clinical articles, including prospective randomized studies of cancer immunotherapy, to publish. CIIM will continue as a key global platform, featuring innovative and reliable information, as an important reference source for clinical oncologists and cancer researchers. 
As any significant achievements in cancer immunotherapy are dependent on the efforts of several generations of scientists and clinicians, we encourage reviewers and authors to consider that CIIM is not only a vehicle for new knowledge and information, but also a critical potential venue supporting professional development of the authors, especially for young graduate students and fellows. In this regard, we identify the positive aspects of research articles received, their significance and innovation in the current paradigm of the field, along with a thoughtful assessment of how a manuscript could be improved.

As we enter a new era of cancer immunotherapy, although equipped with many state-of-art technologies and swamped with big data, one critical thing we shall recognize, is that any significant discovery achieved is based on previous solid work and science. CIIM will continue to provide the solid science needed to pave a path for more successes in cancer treatment.

Publisher's Note Springer Nature remains neutral with regard to jurisdictional claims in published maps and institutional affiliations. 